\title{
High-Speed Waveguide Integrated Silicon Photodetector on SiN-SOI Platform for Short Reach Datacom
}

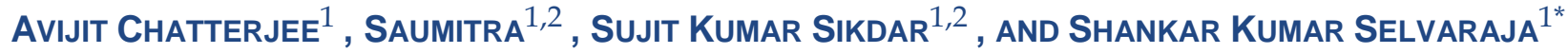 \\ ${ }^{1}$ Center For Nano Science and Engineering, Indian Institute of Science, Bangalore \\ ${ }^{2}$ Molecular Biophysics Unit, Indian Institute of Science, Bangalore \\ ${ }^{*}$ Corresponding author: shankarks@iisc.ac.in
}

We present waveguide integrated high-speed Si photodetector integrated with silicon nitride (SiN) waveguide on SOI platform for short reach data communication in $850 \mathrm{~nm}$ wavelength band. We demonstrate a waveguide couple $\mathrm{Si}$ in photodetector responsivity of $0.44 \mathrm{~A} / \mathrm{W}$ at $25 \mathrm{~V}$ bias. The frequency response of the photodetector is evaluated by coupling of a femtosecond laser source through SiN grating coupler of the integrated photodetector. We estimate a $3 \mathrm{~dB}$ bandwidth of $14 \mathrm{GHz}$ at $20 \mathrm{~V}$ bias, highest reported bandwidth for a waveguide integrated Si photodetector. We also present detailed optoelectronic DC and AC characterisation of the fabricated devices. The demonstrated integrated photodetector could enable an integrated solution for scaling of short reach data communication and connectivity.

Ever increasing data traffic drives the need for scalable, high speed, and energy efficient connectivity in the datacenters [1-3]. Optical connectivity offers an excellent solution to data connectivity bottleneck in datacenters. Widely used $850 \mathrm{~nm}$ VCSEL based Multimode fiber (MMF) technology is reaching maximum capacity, however, proposal to extend the MMF toward Wavelength Division Multiplexing (WDM) would scale up the capacity to $400 \mathrm{Gbps}$. In view of that, IEEE proposed $400 \mathrm{Gbps}$ roadmap [4] for short reach rack-to-rack communication by 2020. Adapting WDM would require integration of wavelength multiplexers and demultiplexers, light sources and detectors in a single module. Integrating all the functional elements as a single photonic integrated circuit would enable a compact, costeffective, and scalable platform.

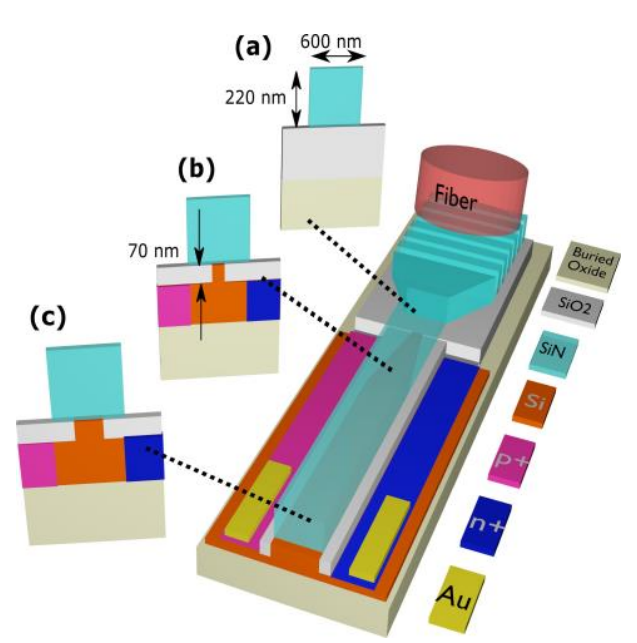

Fig. 1. Schematics of the proposed waveguide coupled Si photodetector. (a) $\mathrm{SiN}$ waveguide on $\mathrm{SiO}_{2}$, (b) Tapered Si waveguide vertically coupled to SiN, and (c) Si pin photodetector with SiN waveguide.

It is essential that the circuit should be able to guide light with low-loss and is compact with the integration of light sources and detectors. Silicon (Si) photonics has demonstrated such a versatile platform in $1310 \mathrm{~nm}$ and $1550 \mathrm{~nm}$ wavelength band [5-7]. A similar strategy could enable a photonic circuit platform in $850 \mathrm{~nm}$ band as well. Recently, efforts are made to integrate III$\mathrm{V}$ light sources and III-V/Graphene photodetectors on Silicon Nitride (SiN) waveguide platform [7, 8]. However, such hybrid integration requires complex assembly and post-processing technology, also the fabrication is not compatible with preferred $\mathrm{Si}$ process technology. It is desirable to reduce such non-standard integration. In this regards, $\mathrm{Si}$ is an excellent photodetector in the $850 \mathrm{~nm}$ band with higher responsivity than III-V photodetector. However, longer absorption length, RC time, and transit time in Si considerably limit bandwidth [9-11]. The bandwidth limitation of Si photodetector is significantly improved by realizing lateral pin photodetector in a guided wave configuration, particularly in a Silicon-on-Insulator (SOI) platform [12]. The thin $\mathrm{Si}$ in SOI has smaller depletion capacitance, which enhances the RC time limited bandwidth.

In this paper, we present $\mathrm{SiN}$ waveguide integrated high- 
speed Si pin photodetector on the SOI platform. Figure 1 illustrates a schematics of the proposed device that consists of $\mathrm{SiN}$ optical waveguide, $\mathrm{SiN}$ grating coupler, $\mathrm{SiN}-\mathrm{Si}$ inverse taper coupler, and Si pin photodetector. For optical waveguide, $\mathrm{SiN}$ is an attractive CMOS compatible material that has large transparency window; visible to mid-IR wavelength band and explored widely for the linear and non-linear properties. $[8,13]$

The light from an optical fiber is coupled into the chip using a grating fiber coupling in the SiN waveguide. The waveguide dimensions were optimized to support single mode operation with a waveguide width and thickness of $600 \mathrm{~nm}$ wide and 220 $\mathrm{nm}$ respectively. To couple $850 \mathrm{~nm}$ light from a fiber into the $\mathrm{SiN}$ waveguide, grating coupler and linear taper are used. An iterative optimization of the grating parameters such as grating period, etch depth and fill-factor were done to achieve maximum coupling efficiency. From the FDTD simulation, a coupling efficiency of $9.6 \mathrm{~dB} /$ coupler with a $1 \mathrm{~dB}$ bandwidth of $12 \mathrm{~nm}$ for air-clad SiN grating couplers is achieved with a grating period of $650 \mathrm{~nm}$ and etch depth of $180 \mathrm{~nm}$. The grating performance was experimentally verified by fabricating gratings in $220 \mathrm{~nm}$ thick LPCVD SiN over $2.2 \mu \mathrm{m}$ thick $\mathrm{SiO}_{2}$. Figure 2 (a) and (b) shows the cross-section of the fabricated $\mathrm{SiN}$ grating coupler and SEM image of the SiN waveguide and grating coupler respectively. AFM image of the grating coupler is shown in Figure 2 (c) and (d) shows the etch depth of around $180 \mathrm{~nm}$. The coupling efficiency is characterized using a fiber-coupled LED source (850$900 \mathrm{~nm}$ ) and optical spectrum analyzer. Figure 2 (e) shows the measured coupling efficiency of a SiN grating coupler with 650 $\mathrm{nm}$ grating period yielding a maximum coupling efficiency of $12.6 \mathrm{~dB} /$ coupler around $867 \mathrm{~nm}$.
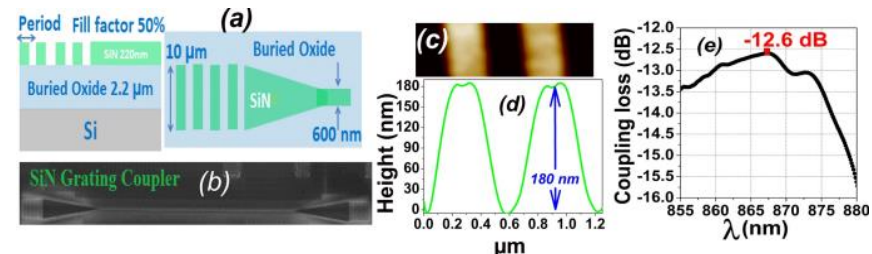

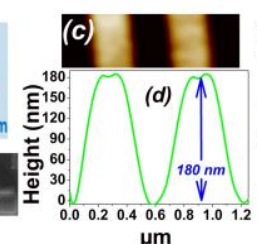

Fig. 2. (a) Schematics of the test structure (top and cross section view), (b) SEM image of the fabricated SiN grating, (c) AFM image of the grating, (d) Shows the etch depth of $180 \mathrm{~nm}$ of gratings obtained from AFM, (e) Grating coupling efficiency Vs wavelength

The propagating light in the SiN layer should be coupled into the underlying Si waveguide photodetector layer. In the proposed structure, the evanescent mode coupling is used to couple light between the SiN and Si layer. Due to a large mode mismatch difference in the two waveguides, the vertical coupler requires a longer length to efficiently couple light from SiN to $\mathrm{Si}$ waveguide. However, longer coupling length would increase the Si photodetector length thus degrades the $3 \mathrm{~dB}$ bandwidth due to higher depletion capacitance [14]. To achieve higher coupling efficiency with a large bandwidth of the photodetector, we introduce Si inverse taper as illustrated in Figure 1. Si taper helps to achieve higher coupling efficiency between $\mathrm{SiN}$ and Si with shorter overlapping coupler length due to better phase matching. Shorter overlapping length enhances the bandwidth of the photodetector as well. To optimize the geometry of the Si inverse taper, we use commercial simulation software Fimmprop [15] and Lumerical FDTD [16]. Figure 3a shows cross-section beam propagation and mode coupling from the top SiN layer into Si layer. Successive optimization of the vertical direction coupler length and taper parameters were performed to achieve maximum coupling. Figure $3 \mathrm{~b}$ shows the simulated coupling efficiency versus taper length $\left(L_{T}\right)$ at $850 \mathrm{~nm}$ wavelength for optimized silicon taper as described in Figure 3c. An optimised coupling efficiency of $72 \%$ is obtained from the simulation for a 15 $\mu m$ long Si taper. The coupling efficiency could be increased by making the longer direction coupler, however, this could result in lower bandwidth. Therefore, Si taper length of $15 \mu \mathrm{m}$ is optimum to achieve high responsivity as well as large bandwidth of the photodetector. Si absorbs the coupled light in the $850 \mathrm{~nm}$ wavelength band and generates Electron and Hole Pairs (EHPs). The generated EHPs are collected laterally by the photodetector along the propagation direction. Since pin photodetector has larger absorption region and lower depletion capacitance, we have chosen pin over $p n$ junction photodetector. Moreover, the shallowly etched ridge geometry of the Si photodetector confines the coupled light in the intrinsic region which minimises the photocarrier generation outside the depletion region. Photocarriers outside depletion region must diffuse before getting collected, which limits the bandwidth of the photodetector. Hence, ridge waveguide helps to improve the photodetector bandwidth further. Furthermore, to achieve the highest bandwidth, intrinsic width of the pin needs to be optimised. From device simulations, intrinsic width of $200 \mathrm{~nm}$ for $15 \mu \mathrm{m}$ long pin photodetector gave a maximum $3 \mathrm{~dB}$ bandwidth of $25 \mathrm{GHz}$.

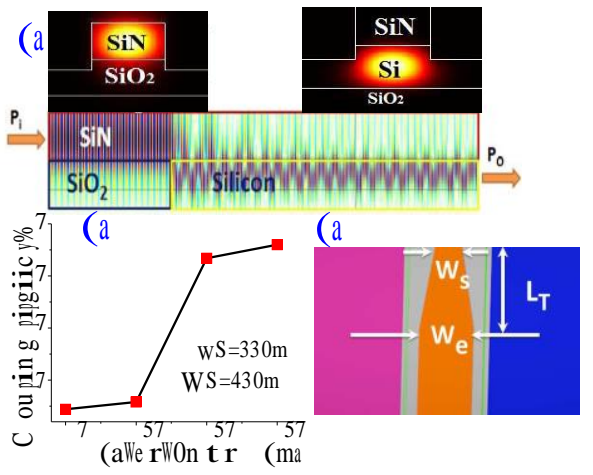

Fig. 3. (a) Optical simulation of $\mathrm{Si}$ and $\mathrm{SiN}$ waveguide and coupler, (b) Coupling efficiency from SiN waveguide into $\mathrm{Si}\left(P_{O} / P i\right)$ for different Silicon taper length $\left(L_{T}\right)$, and (c) Top View of the Si taper configuration.

The optimized devices were fabricated on a photonics SOI substrate having $220 \mathrm{~nm}$ thick Si device layer on $2 \mu \mathrm{m}$ thick buried oxides. Figure 4 depicts an overview of the fabrication process flow. The photodetector structure in the Si layer was patterned by using optical lithography followed by dry etching. The Si pattern was then coved with silicon dioxide and planarized using CMP. The doping of $p+$ and $n+$ regions were realized using diffusion doping process. The doping windows were placed $4 \mu \mathrm{m}$ apart to avoid overlapping of the dopants during diffusion doping process [17]. To form the SiN waveguide and grating coupler, LPCVD SiN was deposited and patterned using e-beam lithography, and dry etch process. Before SIN deposition $50 \mathrm{~nm} \mathrm{SiO} 2$ is deposited as a liner layer to project the underlying Si during SiN pattering. Finally, Chrome/Gold electrical contacts were made on $p+$ and $n+$ dopant window. Figure 5 shows SEM image of the fabricated device. A set of three devices with varying Si taper parameter were fabricated. Figure 5 shows the set of devices (D1, D2 and D3) that were fabricated. All the devices were designed with a detector length of $100 \mu \mathrm{m}$. Since the junction starts from the taper, the effective detector length is $L_{T}+100 \mu m$.

Experimental validation of the fabricated device was performed by measuring responsivity and bandwidth of the pho- 


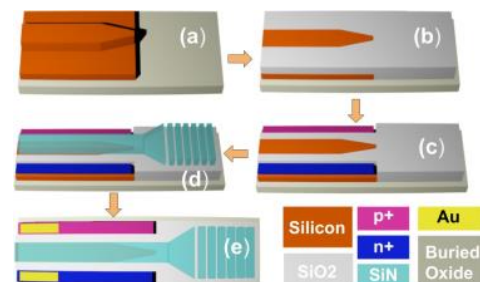

Fig. 4. Fabrication process IIOW (a) sillcon pattering using

optical lithography and dry etching, (b) $\mathrm{SiO}_{2}$ deposition and planarization, (c) p+ and $\mathrm{n}+$ junction region defined using diffusion doping, (d) $220 \mathrm{~nm}$ LPCVD SiN deposited and patterned (waveguide and grating coupler) using e-beam lithography and dry etching, and (e) Cr-Au deposited by e-beam evaporator followed by lift-off to define electrical contacts.

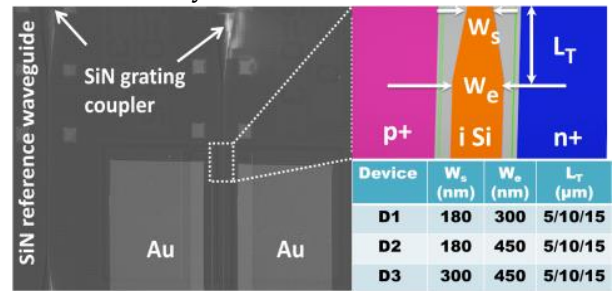

Fig. 5. Scanning electron microscope image of the fabricated device (left) and Taper parameters of Device D1, D2, and D3 fabricated (device total length $\left.=L_{T}+100 \mu \mathrm{m}\right)$, intrinsic width $=$ $4 \mu m$ (right).

todetector. Initial DC characterization is performed to determine the dark current and responsivity. Light is coupled into the chip using a grating coupler as mentioned earlier. For responsivity measurement, the optical power delivered into the waveguide is measured using a reference $\mathrm{SiN}$ waveguide with in and out coupler. Figure 6 (a) and (b) show the measured dark current and photocurrent of the fabricated devices (D1, D2 and D3) each with $115 \mu m$ length. All the device configurations measured a photocurrent that is two orders of magnitude higher than the dark current. We measure a dark current as low as $46 \mathrm{nA}$ at 10 $\mathrm{V}$ bias, at the same voltage we measure a photocurrent of 5.09 $\mu A$ for device D3. We measure the highest photocurrent of 7.5 $\mu A$ at $25 \mathrm{~V}$ for device D3. At higher bias voltage photocurrent increases due to impact ionization. From Figure 6(b) we observe that the photocurrent saturates beyond $15 \mathrm{~V}$ for device D3. This occurs due to space charge effect which reduces the electric field at the center of the pin [12]. This effect is more prominent for device D3 than D1 and D2 since D3 has higher dark current than D1 and D2 at higher bias voltages.
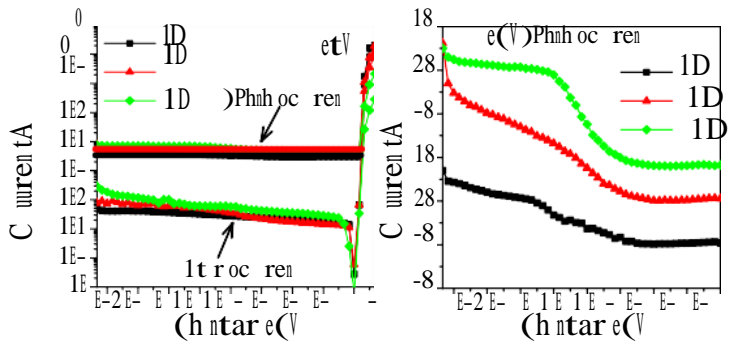

Fig. 6. IV characteristics of photodetector for different devices (D1-D3) of length $\mathrm{L}=115 \mu m$, (a) Log scale, (b) Linear scale

The responsivity of the detector is measured from the DC photocurrent and power coupled into the waveguide. Figure 7 shows the summary of the responsivity of the fabricated detectors. Irrespective of the Si-SiN taper design, detector with longest taper length of $15 \mu \mathrm{m}$ measured maximum responsivity in each design set. As the length of the total device increases, the responsivity increases due to better power coupling between SiN and Si waveguide. However, responsivity for different devices (D1-D3) are almost identical up to $10 \mathrm{~V}$ bias due to insignificant change in absorption region. Beyond $10 \mathrm{~V}$ bias, responsivity of (D1-D3) starts to deviate and shows increase in responsivity with bias due to impact ionization effect. we measure a maximum responsivity of $0.44 \mathrm{~A} / \mathrm{W}$ at $25 \mathrm{~V}$ for device $\mathrm{D} 3$.
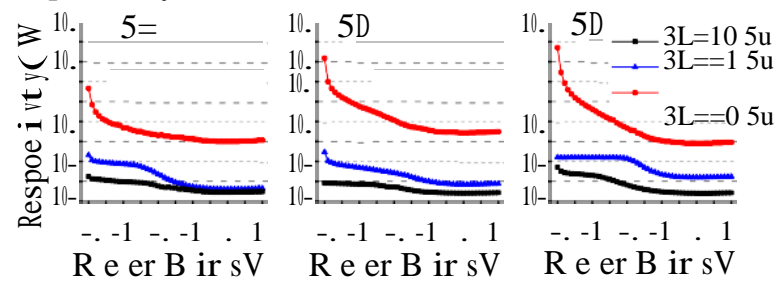

Fig. 7. Responsivity of devices D1, D2, D3 for different length of $\mathrm{Si}$ photodetector at various bias voltage.

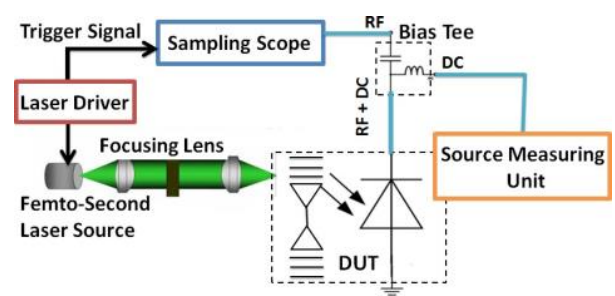

Fig. 8. Frequency response measurement setup with femtosecond pulse Laser.

The frequency response of the detector is measured using a femtosecond pulsed laser source [18]. The $800 \mathrm{~nm}$ femtosecond laser source generates a pulse of width $280 \mathrm{fs}, 200 \mathrm{~mW}$ of average power at $80 \mathrm{MHz}$ repetition rate. Figure 8 shows a schematic of the experimental setup used to measure the pulse response of the detector. Generated current pulse output is fed into the Bias Tee (26 GHz cut off) to separate the AC and the DC components. DC component is measured by a source measuring unit. The AC component of the pulse output is measured using a WideBandwidth Oscilloscope (DCA-X 86100D).

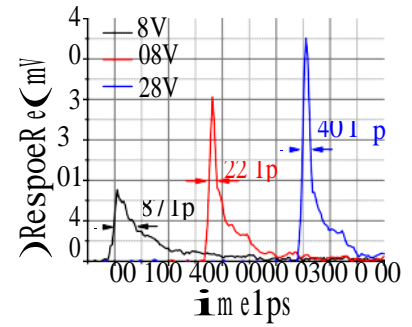

Fig. 9. Pulse response of the photodetector (device D3) at different reverse biases.

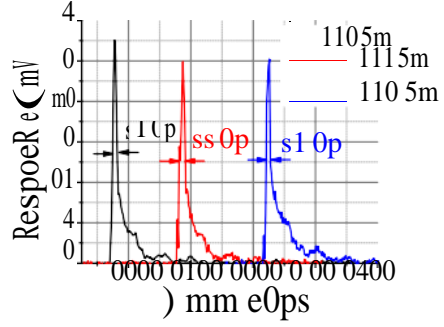

Fig. 10. Pulse response of photodetector for different lengths of device $\mathrm{D} 3$ at $20 \mathrm{~V}$ bias.
Similar to DC measurement, we measured both waveguide coupled and direct illumination frequency response. The femtosecond source of spot size $5 \mu \mathrm{m}$ is illuminated on top of the intrinsic region to evaluate the frequency response of the de-
tector. Figure 9 shows the temporal pulse response of device

D3 with $105 \mu m$ length for different bias voltages. The $3 \mathrm{~dB}$ frequency $\left(f_{3 d B}\right)$ of the photodetector is estimated from the full width at half maximum (FWHM) of the current pulse by the relation $f_{3 d B}=\frac{0.45}{F W H M}[19]$. We measured the best FWHM of 29 ps corresponds to the $3 \mathrm{~dB}$ bandwidth of $15.5 \mathrm{GHz}$ at $10 \mathrm{~V}$ reverse bias. FWHM at $0 \mathrm{~V}$ is wider than at $10 \mathrm{~V}$ due to the longer transit time of the carriers at lower bias voltages. However, with an increase in the bias voltage to reduce transit time could result in avalanche subsequently reducing the detector speed. Figure 9 depicts the evolution of the photodetector response with varying bias. Figure 10 shows the pulse output for different lengths 
of the photodetector. FWHM does not vary significantly with the change in device length that suggests detector bandwidth is not RC time-limited rather transit time limited. To confirm transit time limitation, depletion capacitance, contact pad resistance, and diode series resistance of the pin were measured. Depletion capacitance of the pin is estimated from the relation $C=\frac{e_{0} e_{S i} A}{W_{S i}}$, where $A$ is the cross-section area of the photodetector $(100 \mu m \times 220 \mathrm{~nm})$ and $W_{S i}(3 \mu m)$ is the intrinsic width of the pin which is derived by subtracting the lateral diffusion length of dopant from the intrinsic width designed in lithography $(4 \mu \mathrm{m})$. Calculated capacitance for the pin is extremely low ( $0.76 \mathrm{fF})$. Calculated Contact pad capacitance is in the order of sub fF hence neglected. Contact pad resistance and diode series resistance are obtained from the I-V of the photodiode that comes around $416 \Omega$. It is evident from the low-capacitance and resistance the device bandwidth is transit time limited.

As mentioned earlier, femo-second source is waveguide coupled into the detector through the grating coupler. Since the source was placed vertical to the grating coupler instead of the designed angle of $10^{\circ}$, the power coupling was lower, however, sufficient to measure the pulse response. Figure 11 and 12 present the photodetector response obtained by coupling the femtosecond laser through the SiN grating coupler of the integrated photodetector. Figure 11 shows the pulse response for different devices (D1-D3). Figure 12 summarizes the frequency response of device D3 with different device lengths. Changes in device length of the detector does not cause a significant change in frequency response because the detector bandwidth is transit time limited as discussed in the previous section. Best FWHM of 32 ps corresponds to $14 \mathrm{GHz} 3 \mathrm{~dB}$ bandwidth at $20 \mathrm{~V}$ reverse bias is obtained for device D3 with $115 \mu \mathrm{m}$ length. Since femtosecond laser source is illuminated on the SiN gratings at zero degree angle, the coupling efficiency of SiN grating is low. Due to which amplitude of the pulse output at lower reverse bias voltages falls under the noise floor of the measuring instruments.
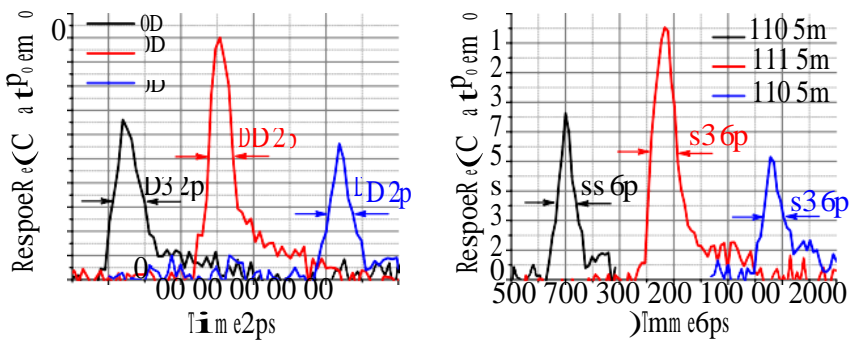

Fig. 11. Pulse response for de- Fig. 12. Effect of device length vices D1-D3 of length $105 \mu m$ (device D3) on pulse response at $20 \mathrm{~V}$ bias with pulse laser at $20 \mathrm{~V}$ bias with pulse laser coupled to $\mathrm{SiN}$ grating coupler. coupled to $\mathrm{SiN}$ grating coupler.

\begin{tabular}{|c|c|c|c|c|c|c|}
\hline Parameters & {$[11]$} & {$[12]$} & {$[14]$} & {$[7]$} & This Work & This Work \\
\hline Dark Current (nA) & 0.06 & $2 \times 10^{3}$ & 0.23 & 22 & 46 & 75 \\
\hline Responsivity (A/W) & - & 0.3 & 0.32 & 0.12 & 0.2 & 0.29 \\
\hline Bandwidth (GHz) & 19.5 & 16.4 & - & 20 & 15.5 & 14 \\
\hline Bias (V) & 5 & 14 & 9 & 2 & 10 & 20 \\
\hline Integrated & No & No & Yes & Yes & No & Yes \\
\hline Platform & Si & SOI & Si & III-V & SOI & SOI \\
\hline
\end{tabular}

Table 1. Comparison with the integrated photodetectors for short reach optical interconnects in the literature.

Table 1 compares the results of this work with previously published works in literature. The bandwidth of $14 \mathrm{GHz}$ and responsivity of $0.29 \mathrm{~A} / \mathrm{W}$ with $75 \mathrm{nA}$ dark current at $20 \mathrm{~V}$ re- verse bias are obtained with $115 \mu m$ long device. To the best of our knowledge, this is the highest reported $3 \mathrm{~dB}$ bandwidth for waveguide integrated Si photodetector.

In conclusion, we have successfully demonstrated an integrated lateral silicon pin photodetector in SiN-on-SOI platform. Silicon pin is realized using simple diffusion doping. SiN waveguides modes are coupled into the intrinsic region by an optimized inverse taper vertical coupler. We obtained the highest responsivity of $0.44 \mathrm{~A} / \mathrm{W}$ at $25 \mathrm{~V}$, and to the best of our knowledge highest $3 \mathrm{~dB}$ cut off frequency of $14 \mathrm{GHz}$ at $20 \mathrm{~V}$ bias for a waveguide integrated silicon photodetector. We find that bandwidth increase with increasing bias voltage reveals that the speed is limited by transit time which can be further improved by the narrower intrinsic width of the pin junction that can be achieved using implantation doping. The demonstration shows a feasible method to integrated standard Si process and $\mathrm{SiN}$ integration to achieved high-speed photodetector operating in short reach wavelength band. The demonstration allows integration of wavelength selective detection that can be used for short reach wavelength division multiplexing.

Acknowledgement. "We thank DST-SERB for funding this research. We also acknowledge funding support from MHRD through NIEIN project, from MeitY and DST through NNetRA"

\section{REFERENCES}

1. D. A. Miller, Proc. IEEE 97, 1166 (2009).

2. M. A. Taubenblatt, J. Light. Technol. 30, 448 (2012).

3. J. A. Tatum, D. Gazula, L. A. Graham, J. K. Guenter, R. H. Johnson, J. King, C. Kocot, G. D. Landry, I. Lyubomirsky, A. N. Maclnnes, E. M. Shaw, K. Balemarthy, R. Shubochkin, D. Vaidya, M. Yan, and F. Tang, J. Light. Technol. 33, 727 (2015).

4. IEEE, "IEEE p802.bs $400 \mathrm{GbE}$ baseline summary," (2015).

5. A. Sobhani, M. W. Knight, Y. Wang, B. Zheng, N. S. King, L. V. Brown, Z. Fang, P. Nordlander, and N. J. Halas, Nat. communications 4, 1643 (2013).

6. M. Casalino, J. Light. Technol. 36, 1766 (2018).

7. G. Chen, J. Goyvaerts, S. Kumari, J. V. Kerrebrouck, M. Muneeb, S. Uvin, Y. Yu, and G. Roelkens, Opt. Express 26, 6351 (2018).

8. Y.Gao, G. Zhou, N. Zhao, H. K. Tsang, and C. Shu, Opt. letters 43, 1399 (2018).

9. S. M. Sze and K. K. Ng, Physics of semiconductor devices (WileyInterscience, 2007).

10. G. Li, K. Maekita, H. Mitsuno, T. Maruyama, and K. liyama, Jpn. J. Appl.

11. P. Gá. 54, H. Cans.06 $(2015)$ G. Polat, S. Ghandiparsi, A. Kaya, H. H. Mam-

taz, A. S. Mayet, Y. Wang, X. Zhang, T. Yamada et al., Nat. Photonics 11, 301 (2017).

12. M. M. Pour Fard, C. Williams, G. Cowan, and O. Liboiron-Ladouceur, Opt. Express 25, 5107 (2017).

13. A. Subramanian, P. Neutens, A. Dhakal, R. Jansen, T. Claes, X. Rottenberg, F. Peyskens, S. Selvaraja, and B. Helin, Philippe Du Bois, IEEE Photonics J 5, 2202809 (2013).

14. A. Samusenko, D. Gandolfi, G. Pucker, T. Chalyan, R. Guider, M. Ghulinyan, and L. Pavesi, J. Light. Technol. 34, 969 (2016).

15. "Optical Propagation Software using EigenMode Expansion (EME) FIMMPROP,"

16. "FDTD Solutions / Lumerical's Nanophotonic FDTD Simulation Software," .

17. V. Jeyaselvan and S. K. Selvaraja, IEEE Photonics Technol. Lett. 30, 2163 (2018).

18. A. Singh, IEEE Photonic Tech L 22, 589 (2010).

19. L. Chen and M. Lipson, Opt. Express 17, 7901 (2009). 


\section{FULL REFERENCES}

1. D. A. Miller, "Device requirements for optical interconnects to silicon chips," Proc. IEEE 97, 1166-1185 (2009).

2. M. A. Taubenblatt, "Optical interconnects for high-performance computing," J. Light. Technol. 30, 448-458 (2012).

3. J. A. Tatum, D. Gazula, L. A. Graham, J. K. Guenter, R. H. Johnson, J. King, C. Kocot, G. D. Landry, I. Lyubomirsky, A. N. Maclnnes, E. M. Shaw, K. Balemarthy, R. Shubochkin, D. Vaidya, M. Yan, and F. Tang, "VCSEL-Based Interconnects for Current and Future Data Centers," J. Light. Technol. 33, 727-732 (2015).

4. IEEE, "IEEE p802.bs $400 \mathrm{GbE}$ baseline summary," (2015).

5. A. Sobhani, M. W. Knight, Y.Wang, B. Zheng, N. S. King, L. V. Brown, Z. Fang, P. Nordlander, and N. J. Halas, "Narrowband photodetection in the near-infrared with a plasmon-induced hot electron device," Nat. communications 4, 1643 (2013).

6. M. Casalino, "Design of resonant cavity-enhanced schottky graphene/silicon photodetectors at $1550 \mathrm{~nm}$," J. Light. Technol. 36, 1766-1774 (2018).

7. G. Chen, J. Goyvaerts, S. Kumari, J. V. Kerrebrouck, M. Muneeb, S. Uvin, Y. Yu, and G. Roelkens, "Integration of high-speed GaAs metalsemiconductor-metal photodetectors by means of transfer printing for $850 \mathrm{~nm}$ wavelength photonic interposers," Opt. Express 26, 6351-6359 (2018).

8. Y. Gao, G. Zhou, N. Zhao, H. K. Tsang, and C. Shu, "High-performance chemical vapor deposited graphene-on-silicon nitride waveguide photodetectors," Opt. letters 43, 1399-1402 (2018).

9. S. M. Sze and K. K. Ng, Physics of semiconductor devices (WileyInterscience, 2007).

10. G. Li, K. Maekita, H. Mitsuno, T. Maruyama, and K. liyama, "Over 10 $\mathrm{GHz}$ lateral silicon photodetector fabricated on silicon-on-insulator substrate by CMOS-compatible process," Jpn. J. Appl. Phys. 54, 04DG06 (2015).

11. Y. Gao, H. Cansizoglu, K. G. Polat, S. Ghandiparsi, A. Kaya, H. H. Mamtaz, A. S. Mayet, Y. Wang, X. Zhang, T. Yamada et al., "Photon-trapping microstructures enable high-speed high-efficiency silicon photodiodes," Nat. Photonics 11, 301 (2017).

12. M. M. Pour Fard, C. Williams, G. Cowan, and O. Liboiron-Ladouceur, "High-speed grating-assisted all-silicon photodetectors for $850 \mathrm{~nm}$ applications," Opt. Express 25, 5107 (2017).

13. A. Subramanian, P. Neutens, A. Dhakal, R. Jansen, T. Claes, X. Rottenberg, F. Peyskens, S. Selvaraja, and B. Helin, Philippe Du Bois, "Low-loss single mode pecvd silicon nitride photonic wire waveguides for 532-900 nm wavelength window fabricated within a cmos pilot line," IEEE Photonics J 5, 2202809-2202809 (2013).

14. A. Samusenko, D. Gandolfi, G. Pucker, T. Chalyan, R. Guider, M. Ghulinyan, and L. Pavesi, "A SiON microring resonator-based platform for biosensing at $850 \mathrm{~nm}$," J. Light. Technol. 34, 969-977 (2016).

15. "Optical Propagation Software using EigenMode Expansion (EME) FIMMPROP," .

16. "FDTD Solutions | Lumerical's Nanophotonic FDTD Simulation Software," .

17. V. Jeyaselvan and S. K. Selvaraja, "Lateral dopant diffusion length measurements using silicon microring resonators," IEEE Photonics Technol. Lett. 30, 2163-2166 (2018).

18. A. Singh, "Impact of Optical Pulsewidth on the Frequency Response of P-i-N Photodiodes," IEEE Photonic Tech L 22, 589-591 (2010).

19. L. Chen and M. Lipson, "Ultra-low capacitance and high speed germanium photodetectors on silicon," Opt. Express 17, 7901-7906 (2009). 